\title{
Probe-Sample Interaction in Aperture-type THz Near-Field Microscopy of Complementary Resonators
}

\author{
Lucy Hale ${ }^{1}$, Janine Keller ${ }^{2}$, Tom Siday ${ }^{1}$, Rodolfo I. Hermans ${ }^{1}$, Johannes Haase ${ }^{3}$, John L. Reno ${ }^{4}$, \\ Igal Brener ${ }^{4}$, Giacomo Scalari ${ }^{2}$, Jérôme Faist ${ }^{2}$, and Oleg Mitrofanov ${ }^{1,4}$ \\ ${ }^{1}$ Electronic and Electrical Engineering, University College London, London WC1E 7JE, UK \\ ${ }^{2}$ Institute of Quantum Electronics, ETH Zürich, Zürich 8093, Switzerland \\ ${ }^{3}$ Paul Scherrer Institute, Villigen 5232, Switzerland \\ ${ }^{4}$ Sandia National Laboratories, Albuquerque, New Mexico
}

\begin{abstract}
Subwavelength complementary metallic resonators operating in the terahertz $(\mathrm{THz})$ regime are investigated with aperture near-field microscopy and spectroscopy. In contrast to far-field methods, the spectra of individual isolated resonators can be retrieved. We find that we can experimentally gain spectral information without modifying the spectral properties of the resonator with the aperture-type near-field probe by operating it at a separation distance greater than $10 \mu \mathrm{m}$.
\end{abstract}

\section{INTRODUCTION}

$\mathrm{S}$ UBWAVELENGTH metallic resonator structures are instrumental for increasing the interaction of light with matter through tight confinement of the electromagnetic field. In the $\mathrm{THz}$ range, these structures were used to reach the strong and even ultrastrong coupling regimes [1]. In order to study these devices, far-field spectroscopy is typically used due to the relative simplicity and ubiquity of these systems. This is effective when studying arrays, as the contribution of multiple elements results in an increased signal to noise ratio the scattering efficiency of single subwavelength resonators is often too weak to detect with far-field systems. However, inter-resonator coupling in arrays may modify the spectral response [2]. As an alternative, it is possible to use near-field methods, for which the signal strength is not dependent on scattering efficiency into the far-field. In particular, both arrays and isolated devices can be investigated with aperture near-field microscopy. In this technique, the field is probed in a subwavelength-sized region, which is defined by the aperture size. As a result, single resonators and arrays can be investigated with comparable signal-to-noise ratio. In addition to this, spectral amplitude measured in the near-field gives direct information about the strength of a resonance, and unlike for far-field methods, it is not dependent on the size of the array.

A drawback of near-field methods however is that the interaction of the near-field probe with the sample can modify its spectral response. Both the spectral amplitude and frequency of a resonance can be affected by the presence of the near-field probe [3]. Therefore, it is necessary to characterise this interaction and account for this when analyzing results. Here we investigate the probe-sample interaction for complimentary plasmonic resonators and aperture-type $\mathrm{THz}$ near-field probes, for varying separation between them. We show that by maintaining a probe-sample separation beyond a certain threshold, the effect of probesample interaction can be practically eliminated, whilst the measurement still provides spectral information with a high signal-to-noise ratio. We make direct comparison of experimental near-field results with numerical simulations for different probe-sample separation, and we find excellent agreement.

\section{RESULTS}

The aperture near-field system is based on a transmission $\mathrm{THz}$ time domain spectroscopy system, where the sample is illuminated through the substrate by a focused $\mathrm{THz}$ beam [4]. The $\mathrm{THz}$ electric field is analyzed using a near-field probe which consists of a subwavelength metallic aperture integrated with a photoconductive antenna detector directly behind [4]. By placing the near-field probe several micrometers from the surface, evanescent fields on the sample can be probed at a spatial resolution defined by the aperture size. Apertures as
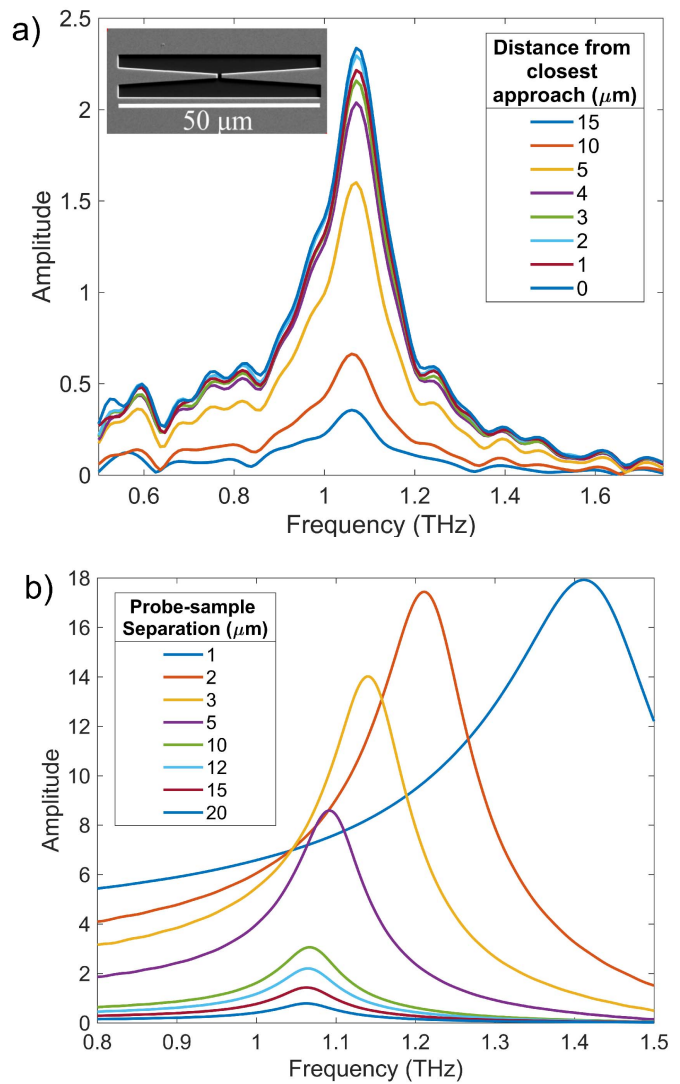

Fig 1. a) Experimentally measured Fourier transform spectra of a single resonator for varying probe-sample separation; the inset shows an SEM image of resonator. b) Simulated Fourier transform spectra of a single resonator for varying probe-sample separation. 
small as $2 \mu \mathrm{m}$ have been successfully used in aperture studies [4]. For all the measurements here a $10 \mu \mathrm{m}$ aperture is used.

Complementary hybrid LC resonators (shown in Fig.1a inset) operating in the $\mathrm{THz}$ regime $(1.1 \mathrm{THz})$ are investigated. The design is a modified version of a nanogap hybrid LC microcavity, which was used to achieve ultrastrong lightmatter coupling at $300 \mathrm{GHz}$ [1]. Resonators are defined by cavities in a $200 \mathrm{~nm}$ gold sheet on a GaAs substrate, and contain a central region of extremely reduced dimensions (1 $\mu \mathrm{m})$ with respect to the illumination wavelength. Here they are investigated with no active medium.

The spectra of a single isolated resonator are experimentally measured for varying probe-sample separations (Fig.1a). It is observed that a clear resonance peak is seen as far as $15 \mu \mathrm{m}$ away from the closest probe-sample separation (where the probe visibly knocks the sample out of alignment). In comparison, we also simulate the full resonator structure with the metallic aperture plane placed at increasing distances away from the sample surface (Fig.1b). As in the experiment, the field is probed behind the aperture. By comparing the experimental and simulated spectra, it can be seen that in both cases, the resonance peak increases significantly as the probe approaches the sample. In the simulated spectra, the spectral frequency stays constant for larger separations, whereas for separations closer than $5 \mu \mathrm{m}$, a larger frequency shift is observed. In contrast, this extreme shift is not seen experimentally.
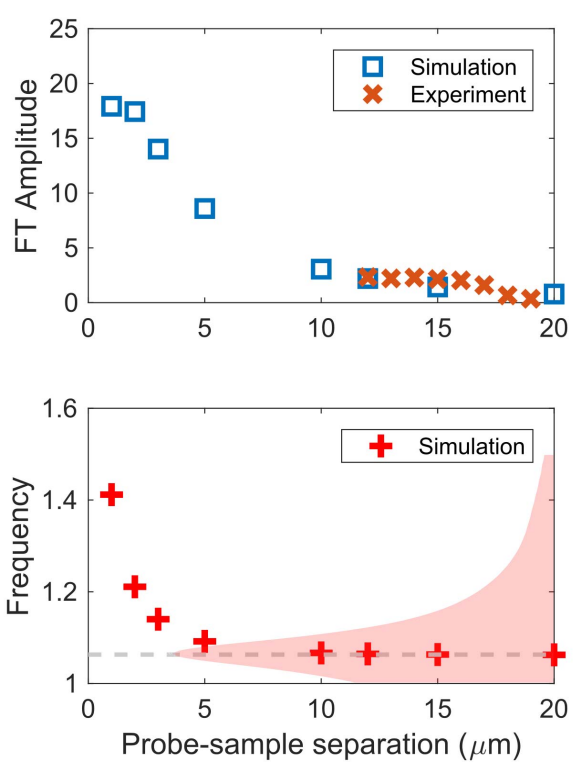

Fig 2 a) Peak simulated FT amplitude with experimental peak FT amplitude fitted b) Simulated peak FT frequency (with spectral curve overlay for comparison to linewidth).

The probe-sample separation is not directly measured in the experiment. However, it can be estimated by comparing the rate of change of spectral amplitude in Fig.1a and Fig.1b. By fitting the experimental Fourier transform amplitudes to the simulated change in amplitude (Fig.2a), we can estimate that the probe-sample separation is greater than $10 \mu \mathrm{m}$. Figure $2 \mathrm{~b}$ shows the simulated change in peak spectral frequency in comparison to the spectral linewidth. At probe-sample separations matching our experiment $(10-20 \mu \mathrm{m})$, the spectral frequency is not altered significantly in comparison to the spectral linewidth. Therefore, we can accurately measure the unperturbed spectral frequency of single complementary resonators by maintaining a probe-sample separation within this range.

\section{SUMMARY}

We demonstrate aperture near-field microscopy of isolated, subwavelength-sized complementary resonators with extremely confined electromagnetic fields. We find a probesample separation range where we can retrieve high signal-tonoise spectroscopic information without affecting their spectral properties. This paves the way for studying strong light-matter coupling at $\mathrm{THz}$ frequencies in the single resonator regime, where inter-resonator coupling does not affect the pure spectral signature.

\section{REFERENCES}

[1] Keller, J., Scalari, G., Cibella, S., Maissen, C., Appugliese, F., Giovine, E., Faist, J. (2017). Nano Letters, 17(12), 7410-7415.

[2] Keller, J., Maissen, C., Haase, J., Paravicini-Bagliani, G. L., Valmorra, F. Palomo, J., Faist, J. Advanced Optical Materials, 5(6), 2017

[3] Khromova, I., Navarro-Cía, M., Brener, I., Reno, J.L., Ponomarev, A., and Mitrofanov, O. Appl. Phys. Lett., 107(2), 21102, 2015.

[4] Mitrofanov, O., Khromova, I., Siday, T., Thompson, R. J., Ponomarev, A N., Brener, I., \& Reno, J. L IEEE Transactions on Terahertz Science and Technology, 6(3), 382-388. 2016.

ACKNOWLEDGEMENTS: This work was supported by the EPSRC (EP/L015277/1, EP/P021859/1, EP/L015455/1) and by the U.S. Department of Energy, Office of Basic Energy Sciences, Division of Materials Sciences and Engineering. JK, JH, JLR, IB, GS, JF acknowledge financial support from the Swiss National Science Foundation (SNF) through the National Centre of Competence in Research Quantum Science and Technology (NCCR QSIT) and Molecular Ultrafast Science and Technology (NCCR MUST). JK, JH, JLR, IB, GS, JF acknowledge financial support from the ERC grant MUSiC. Fabrication of $\mathrm{THz}$ near-field probes were performed at the Center for Integrated Nanotechnologies, an Office of Science User Facility operated for the U.S. Department of Energy (DOE) Office of Science. Sandia National Laboratories is a multimission laboratory managed and operated by National Technology and Engineering Solutions of Sandia, LLC., a wholly owned subsidiary of Honeywell International, Inc., for the U.S. Department of Energy's National Nuclear Security Administration under contract DE-NA0003525 . 